\title{
Scintillation particle detection based on microfluidics
}

\author{
A. Mapelli ${ }^{\mathrm{a}, \mathrm{b}, *}$, B. Gorini ${ }^{\mathrm{b}}$, M. Haguenauer $^{\mathrm{c}}$, S. Jiguet $^{\mathrm{d}}$, G. Lehmann Miotto $^{\mathrm{b}}$, \\ W. Vandelli ${ }^{\mathrm{b}}$, N. Vico Triviño ${ }^{\mathrm{a}}$, P. Renaud ${ }^{\mathrm{a}}$ \\ a Laboratoire de Microsystémes, Ecole Polytechnique Fédérale de Lausanne, EPFL Switzerland \\ ${ }^{\mathrm{b}}$ Physics Department, CERN, Geneva, Switzerland \\ ${ }^{\mathrm{c}}$ Ecole Polytechnique, CNRS/IN2P3, Palaiseau, France \\ d Gersteltec Sàrl, Pully, Switzerland
}

\section{A R T I C L E I N F O}

\section{Article history:}

Received 30 September 2009

Received in revised form 3 March 2010

Accepted 28 March 2010

Available online 8 April 2010

\section{Keywords:}

Particle detectors

Liquid scintillation

Microfluidics

Microfabrication

SU-8 negative photoresist

\begin{abstract}
A B S T R A C T
A novel type of particle detector based on scintillation, with precise spatial resolution and high radiation hardness, is being studied. It consists of a single microfluidic channel filled with a liquid scintillator and is designed to define an array of scintillating waveguides each independently coupled to a photodetector. Prototype detectors built using an SU-8 epoxy resin have been tested with electrons from a radioactive source. The experimental results show a light yield compatible with the theoretical expectations and confirm the validity of the approach.
\end{abstract}

(c) 2010 Elsevier B.V. All rights reserved.

\section{Introduction}

A novel type of particle detector based on capillaries filled with liquid scintillators is being studied. It is possible with microfabrication technologies to build microfluidic devices with dimensional resolutions in the order of $\mu \mathrm{m}$ with a single photolithographic step. Such devices allow the easy manipulation of fluids inside capillaries overcoming the difficulties encountered with previous high spatial resolution liquid scintillation detectors made of capillary bundles [1-4]. Moreover, the possibility to circulate and renew the liquid scintillator makes the active medium of the detector intrinsically radiation hard and by changing the type of scintillator in the microchannels the same device can be used for different types of measurements. Microfabricated devices have been developed and studied to demonstrate experimentally the feasibility of such a detector based on microfluidics. Results obtained with a first series of prototypes are reported elsewhere [5]. This paper describes the results of the experimental investigation performed on the second generation prototype that demonstrates the validity of the approach.

\footnotetext{
* Corresponding author at: EPFL, 1015 Lausanne, Vaud, Switzerland. Tel.: +412169367 45; fax: +41216935950.

E-mail address: alessandro.mapelli@epfl.ch (A. Mapelli).
}

\section{Microfluidic scintillation detector}

The main characteristics of the particle detector investigated are high spatial resolution for precise reconstruction of particle tracks, high radiation resistance to operate in very high radiation environments and low material budget to interfere as least as possible with the particles. Microfabrication and microfluidic technologies are appealing to develop such detectors. With a simple process it is possible to fabricate a single microfluidic channel defining a dense array of optically independent scintillating capillaries with dedicated photodetectors (Fig. 1a). The design is based on the assumption that there is no light transmission between the different capillaries due to the right angles being necessary for fluidic circulation at the end of the straight sections. Fluidic operation of the single microchannel is simple and liquid scintillators can be circulated and renewed. To demonstrate the concept of microfluidic scintillation detection, different prototype detectors have been fabricated and characterized.

\section{Prototype detectors}

The design of the prototype devices defines a detection zone, where impinging particles are detected, with high spatial resolution. $50 \mu \mathrm{m}$ wide waveguides are separated by $10 \mu \mathrm{m}$ wide SU-8 structures on a total length of $1 \mathrm{~cm}$. In this region the microchannels are straight and parallel. They then fan-out from a pitch of $60 \mu \mathrm{m}$ to a pitch of $2.3 \mathrm{~mm}$ over $1 \mathrm{~cm}$ to match the inter-pixel dis- 
(a)

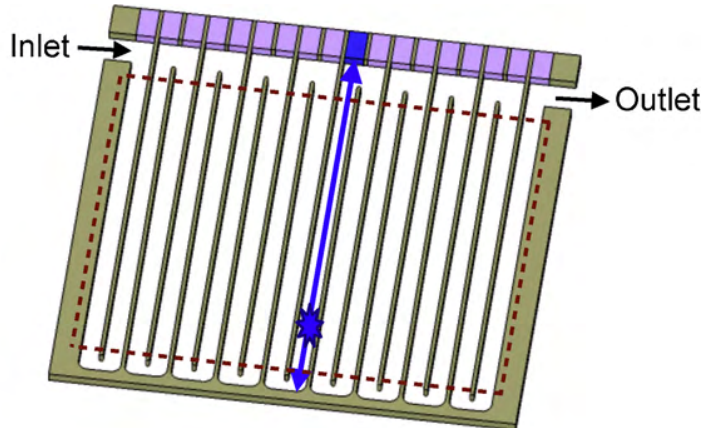

(b)

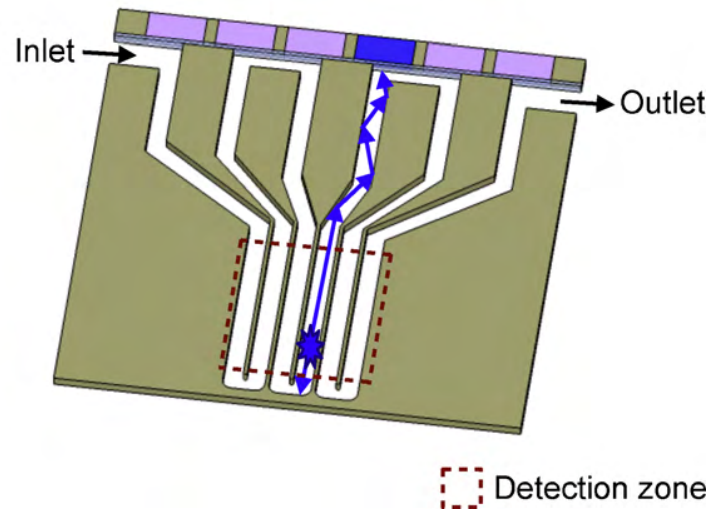

Fig. 1. 3D schematic representation, not to scale, of (a) the principle of operation of the microfluidic scintillation detector and of $(b)$ the fabricated prototype detector A single microfluidic channel defines an array of optically separated waveguides. When a particle interacts with the liquid scintillator in one of the branches the scintillation light is guided towards the corresponding photodetector. In the ideal case (a) all the area covered by the microchannel is used for detection (dotted contour). The layout of the prototype detector (b) has a reduced detection zone (dotted contour) to cope with the pitch of the photodetector available in the experimental test bench.

tance of the multi-anode photomultiplier tube (MAPMT H7546B by Hamamatsu) of the experimental test bench (Figs. 1b and 2).

The microchannels are fabricated by photolithography of the SU-8 resin. This photoresist is commonly used for MEMS [6] and

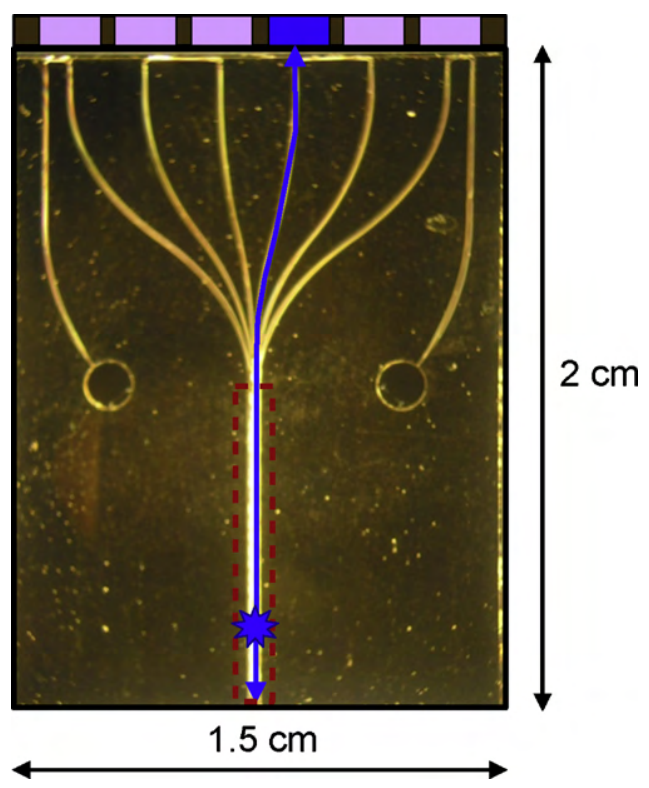

Fig. 2. Top view of the open metallized SU- 8 channels of the prototype microfluidic scintillation detector. The upper side of the chip, in contact with the photodetector pixels, is not metallized to allow light transmission between the microchannels and the photodetector.

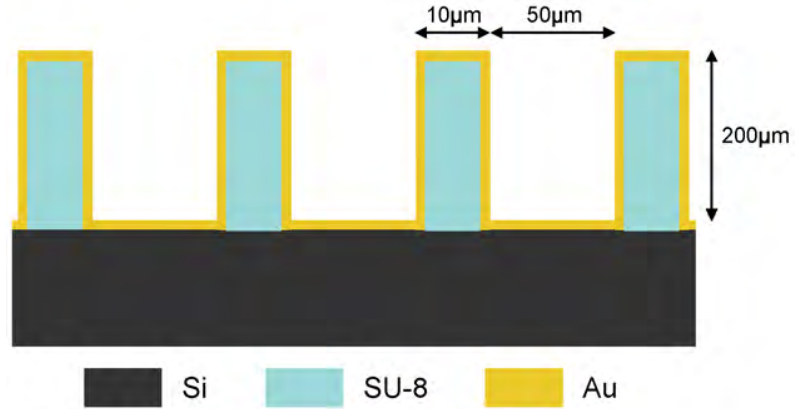

Fig. 3. Schematic representation of the cross-section of the microchannels at the level of the detection zone (not to scale). $200 \mu \mathrm{m}$ thick SU-8 structures $(10 \mu \mathrm{m}$ wide) are separated by $50 \mu \mathrm{m}$ on a silicon substrate. A layer of Au is deposited by sputtering.

microfluidic devices [7] but also for the fabrication of micro-pattern gas detectors [8] and X-ray imagers [9]. The SU-8 resin exhibits outstanding properties such as good adhesion on different types of substrates, high mechanical strength and chemical stability. Of particular interest for this project is the high aspect ratio (1:20) that can be achieved on thick layers in the order of $200 \mu \mathrm{m}$ (Figs. 3 and 4 ) with low roughness vertical sidewalls [10]. Moreover, its high level of resistance to radiation damage, comparable to Kapton film, makes it a good candidate for novel microfabricated radiation detectors [11].

\section{Fabrication of metallized SU-8 microchannels}

The fabrication of the microchannels starts with the spin coating of $200 \mu \mathrm{m}$ thick homogeneous layers of SU-8 (GM1075 from Gersteltec) on silicon wafers ( $100 \mathrm{~s}$ at $950 \mathrm{rpm}$ ), followed by a soft bake $\left(10 \mathrm{~min}\right.$ at $\left.120^{\circ} \mathrm{C}\right)$ and a slow cooling down $\left(4^{\circ} \mathrm{C} / \mathrm{min}\right)$ to avoid the formation of cracks. The coated substrates are then exposed to UV light at a dose of $500 \mathrm{~mJ} / \mathrm{cm}^{2}$ through a mask to polymerize the desired structures. The exposure is followed by a post exposure bake $\left(1 \mathrm{~h}\right.$ at $\left.95^{\circ} \mathrm{C}\right)$ for the cross-linking of the exposed regions. The non-polymerized resin is then dissolved for $20 \mathrm{~min}$ in propylene glycol methyl ether acetate (PGMEA) revealing the microchannels (Fig. 4).

To prevent optical cross-talk between adjacent microchannels and to increase their optical properties, guaranteeing an efficient light transmission from the interaction point to the photodetector, the walls and the bottom of the channels are metallized. Two coat-

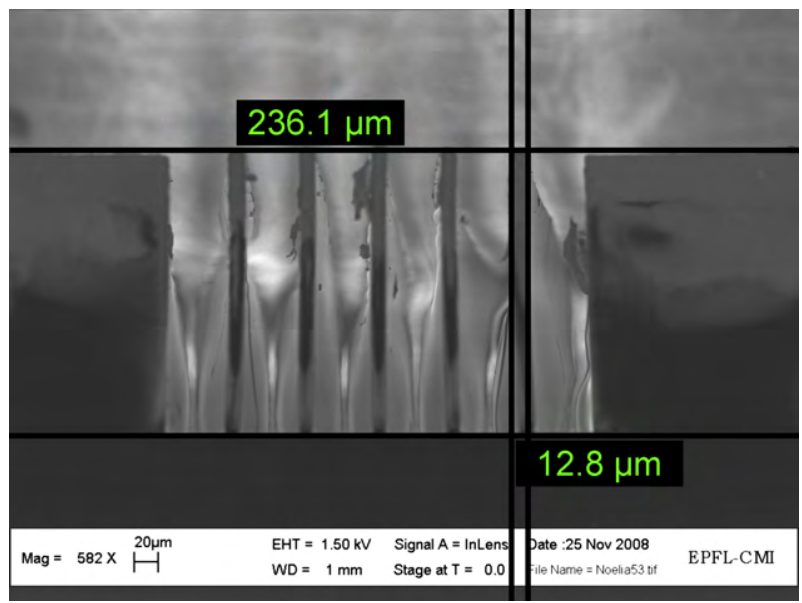

Fig. 4. SEM image of the cross-section of the detection zone of the device. The microchannels are separated by high aspect ratio structures in SU-8. 
ing methods, evaporation and sputtering, have been considered and in both cases an adhesion layer was not necessary. Sputtering gives the best results for our design with high aspect ratio structures. It is performed in the Balzers BAS50. However, due to the high aspect ratio of the walls and their close spacing, metallization is not homogeneous along the wall. It decreases with the depth of the channel. To have sufficient metal at the bottom of the channels and on the lower part of the walls two consecutive metallizations are performed guaranteeing at least $200 \mathrm{~nm}$ of metal at the bottom. The reason to deposit metal in two steps is due to the temperature reached during the sputtering process. A single long deposition results in undulation and collapsing of the thin SU-8 walls while two shorter cycles are not harmful. Au and Al depositions have been performed. The results presented in this paper have been obtained with Au-coated SU-8 microchannels.

At this stage, the wafer is diced to separate 16 chips with dimensions of $1.5 \mathrm{~cm} \times 2 \mathrm{~cm}$ (Fig. 2). They are then individually placed in a mechanical set-up to close the channels for microfluidic manipulation and to optically couple them to the photodetectors. Details of the set-up are given in the following section. The Au coating is removed from the side of the channels coupled to the photodetectors to allow transmission of the scintillation light. Removal is performed by dipping a small portion of the chip in an Au etching solution.

\section{Experimental}

The channel is closed by covering the chip with an Al-coated Mylar foil and encapsulating the whole in a black PMMA block. Optical gel is used to improve the light transmission between the microfluidic chip and the quartz window of the MAPMT. Tubes are connected to the inlet and outlet of the channel through the PMMA block. Filling and circulation of the liquid scintillator in the microchannel is performed with a syringe controlled by hand. In a later stage, and in particular for future beam test experiments, a fluidic circulation controlled with a pump will be implemented. The channels are filled with a liquid scintillator (EJ-305 by Eljen Technology) selected for its high light output (80\% of Anthracene) and for its emission spectrum peaking around $425 \mathrm{~nm}$ in the most sensitive region of the MAPMT. The photoelectric yield of the chipMAPMT assembly is measured by exciting the liquid scintillator with electrons from a collimated ${ }^{90} \mathrm{Sr}$ source which are considered as minimum ionizing particles (MIP). The coincidence of two plastic scintillating fibres (Kuraray SCSF-78 $0.5 \mathrm{~mm}$ square cross-section) placed underneath the detector is used as external trigger on the electrons. For each trigger the signals from the MAPMT channels are sent to a charge-to-digital converter (CAEN QDC V792).

Measurements have also been performed on an independent set-up with a non-pixellated photomultiplier tube (PMT) by masking all the microchannels except the one under test. Results from both test-benches match within the experimental uncertainties mainly due to the optical coupling of the thin microfluidic device orthogonal to the flat window of PMTs.

\section{Results}

The charge spectra of individual scintillating microfluidic channels (200 $\mu \mathrm{m}$ deep and $50 \mu \mathrm{m}$ wide) are fitted with the convolution $F$ (Eq. (1)) of a Poissonian distribution $P$ describing the fluctuations in the production of photoelectrons with Gaussian terms $G$ describing the response of the readout electronics to a given number of photoelectrons [12]:

$F(x) \propto P\left(N, \bar{N}_{p e}\right) \otimes G(x, N, \sigma, \sqrt{N})$

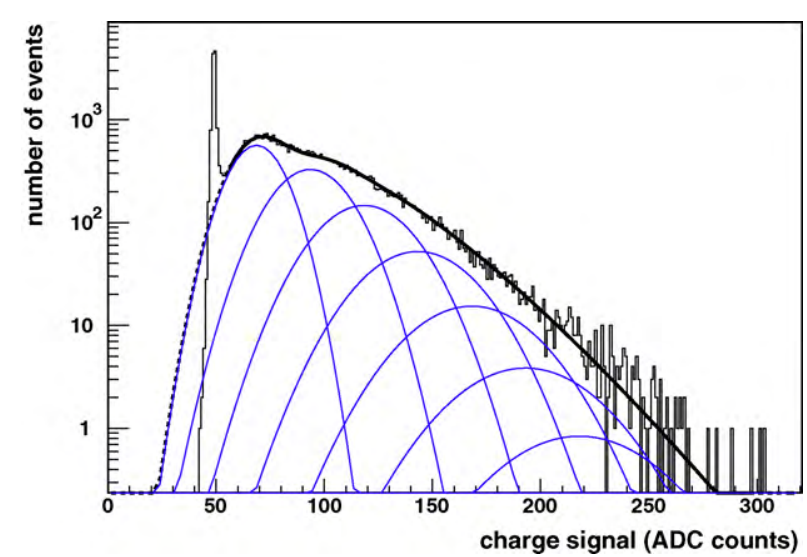

Fig. 5. Photoelectron spectrum obtained by irradiating a $50 \mu \mathrm{m} \times 200 \mu \mathrm{m}$ microchannel with MIPs. The average number of photoelectrons is 1.65 . The blue curves represent the contributions from 1 to 7 photoelectrons and the black curve is the global convolution used to fit the signal. (For interpretation of the references to color in this figure legend, the reader is referred to the web version of the article.)

where $N$ is the number of events, $\bar{N}_{p e}$ is the average number of photoelectrons per event and $\sigma$ is the standard deviation of the distribution of single photoelectron counts. From the fit an average number of photoelectrons $\bar{N}_{p e}$ per incident MIP of 1.65 is derived (Fig. 5).

This number is well in agreement with the theoretical expectations. Assuming that light is guided in the metal-cladded microchannels by attenuated total internal reflection (ATIR) [13] the expected photoelectric yield can be expressed as follows:

$\bar{N}_{p e}=N_{\text {scint }} \cdot \varepsilon_{\text {coll }} \cdot \varepsilon_{\text {refl }} \cdot \varepsilon_{\text {att }} \cdot \varepsilon_{i n} \cdot \varepsilon_{Q_{e f f}}$

where $N_{\text {scint }}$ is the number of scintillation photons produced isotropically in the microchannel by an impinging electron, $\varepsilon_{\text {coll }}$ is the collection efficiency of a rectangular metal-coated microchannel, $\varepsilon_{\text {refl }}$ is the gain due to the reflective end of the channel opposite to the photodetector, $\varepsilon_{a t t}$ is the transport efficiency due to optical absorption in the liquid scintillator, $\varepsilon_{i n}$ is the transmission efficiency at the interface between the microchannel side where Au has been removed and the PMT and $\varepsilon_{Q_{e f f}}$ is the quantum efficiency of the PMT.

A MIP traversing a $200 \mu \mathrm{m}$ channel filled with the liquid scintillator EJ-305 produces a number $N_{\text {scint }}$ of photoelectrons in the order of 420 according to the technical data provided by Eljen Technology. The efficiencies $\varepsilon_{\text {coll }}, \varepsilon_{\text {refl }}$ were estimated to be 0.03 and 1.4 , respectively, by running Monte Carlo simulations. The attenuation length of the liquid scintillator EJ-305 is reported by Eljen Technology to be in the order of $3 \mathrm{~m}$. One can safely assume that there is virtually no attenuation along the $2 \mathrm{~cm}$ long channels and that the transmission efficiency $\varepsilon_{\text {att }}$ is about 0.99 . Around $425 \mathrm{~nm}$, the wavelength of maximum emission of the liquid scintillator, the quantum efficiency of the photomultiplier tube $\varepsilon_{Q_{e f f}}$ is estimated to be about 0.14 [14]. The equation leads to $\bar{N}_{p e} \simeq 1.7$.

A yield of 1.65 photoelectrons leads to a detection efficiency $\varepsilon_{\text {det }}$ up to $80 \%$ as derived from:

$\varepsilon_{\mathrm{det}} \approx 1-P\left(0, \bar{N}_{p e}\right)=1-e^{-1.65}=80.1 \%$

Previous results obtained with microchannels with sharp angles (Fig. 1b) in the path of light transmission showed significantly lower light yield in the order of 0.74 photoelectrons per MIP [5]. This was interpreted as being due to light losses in the angles leading to the current prototype design (Fig. 2). The results obtained with this new prototype are well in agreement with the extrapolation from the previous results. 
The active area of the prototypes covers $83 \%$ of the detection zone. This coverage is dictated by the ratio between the size of the SU-8 structures and the size of the scintillating capillaries. By staggering at least 2 planes a fully active detector can be obtained with small inactive edges. Moreover by staggering 3 layers the device can be self-triggering by requesting a coincidence of 2 planes. The staggering also increases the spatial resolution. A device with 3 staggered planes will be implemented to demonstrate experimentally the increased spatial resolution and the self-triggering capabilities of a fully active detector.

\section{Conclusions}

The working principle of a novel type of scintillation detector based on microfluidics has been demonstrated experimentally. A standard process of UV photopatterning has been optimized to fabricate structures with high aspect ratios (1:20) in thick layers of SU-8 in the order of $200 \mu \mathrm{m}$. They define a dense array of microchannels that are filled with liquid scintillator and optically coupled to the photocathode of an MAPMT. The photoelectric yield of the device measured with MIPs is in the order of 1.65 photoelectrons per MIP for $200 \mu \mathrm{m}$ deep microchannels and is in full agreement with calculations. The high fill factor of the scintillating microchannels, the possibility to measure very close (few $\mu \mathrm{m}$ ) to the edge of the device and the increased radiation hardness makes this novel type of detector particularly interesting for applications such as tracking particles in the field of high energy physics. Moreover, the compact design of microfabricated scintillation detectors opens the way to numerous applications where macrodetectors cannot be used like insitu dosimetry for hadron therapy.

\section{References}

[1] A. Bay, et al., A high-resolution tracking hodoscope based on capillary layer filled with liquid scintillator, Nucl. Instrum. Methods A 457 (2001) 107-116.

[2] RD46 Collaboration, RD46 Status Report-high resolution tracking device based on capillaries filled with liquid scintillator, CERN/LHCC 97-38, 1997.

[3] S. Buontempo, et al., Development of large-volume, high-resolution tracking detectors based on capillaries filled with liquid scintillator, Nucl Instrum. Methods A 360 (1995) 7-12.

[4] A. Artamov, et al., Liquid scintillator calorimetry for the LHC, Nucl. Instrum. Methods A 360 (1995) 240-244.

[5] A. Mapelli, et al., Development and studies of a novel microfabricated radiation hard scintillation particle detector with high spatial resolution, Nucl. Phys. B (Proc. Suppl.) 197 (2009) 43-47.

[6] H. Lorenz, et al., High-aspect-ratio, ultrathick, negative-tone near-UV photoresist and its applications for MEMS, Sens. Actuators A 64 (1998) 33-39.

[7] S. Metz, et al., Polyimide and SU-8 microfluidic devices manufactured by heat depolymerizable sacrificial material technique, Lab Chip 4 (2004) 114-120.

[8] J. Schmitz, Adding functionality to microchips by wafer post-processing, Nucl. Instrum. Methods A 576 (2007) 142-149.

[9] J.H. Daniel, et al., Fabrication of high-aspect-ratio polymer microstructures for large-area electronic portal X-ray images, Sens. Actuators A 140 (2007) 185-193.

[10] R. Guerre, et al., Fabrication of vertical digital silicon optical micromirrors on suspended electrode for guided-wave optical switching applications, Sens. Actuators A 123-124 (2005) 570-583.

[11] M.J. Key, et al., On the radiation tolerance of SU-8, a new material for gaseous microstructure radiation detector fabrication, Rad. Phys. Chem. 71 (2004) 1003-1007.

[12] E.H. Bellamy, et al., Absolute calibration and monitoring of a spectrometric channel using a photomultiplier, Nucl. Instrum. Methods A 339 (1994) 468-476.

[13] R. Guerre, Guided-wave micro-electro-mechanical systems (MEMS) optical switches for telecommunication applications, EPFL Thesis N. 3269, 2005.

[14] S. Ask, et al., Luminosity measurement at ATLAS-development, construction and test of scintillating fibre prototype detectors, Nucl. Instrum. Methods A 568 (2006) 560-588.

\section{Biographies}

Alessandro Mapelli received his MSc in microengineering from the Ecole Poytechnique Fédérale de Lausanne (EPFL), Lausanne, Switzerland in 2005. He has since been working at the European Organization for Particle Physics (CERN) in Geneva, Switzerland for the obtention of a PhD from EPFL. He was involved in the development of a scintillating fibre tracker (ALFA-Absolute Luminosity For ATLAS) to measure the absolute luminosity of the LHC collider at the interaction point of the ATLAS experiment. His work is focused on the development, studies and integration of microsystems to particle detectors.

Bendetto Gorini is a staff in the physics department of the European Organization for Particle Physics (CERN). He holds a PhD in physics from the Scuola Normale Superiore in Pisa, Italy. During his career his fields of interest have ranged from development of experimental techniques to data analysis. In the last few years he was particularly involved in designing and developing the data-acquisition system for the ATLAS experiment on the Large Hadron Collider (LHC). At present he is in charge of the global coordination of the operations of the ATLAS experiment.

Maurice Haguenauer is a physicist at CNRS/IN2P3, Laboratoire Leprince-Ringuet, Ecole Polytechnique, Palaiseau, France after being a staff member of CERN, Geneva, Switzerland until 1987. He obtained his PhD on the CERN Gargamelle neutrino experiment in 1973. He was then involved in forward physics experiments at SPS and now at LHC particle beam colliders. His activities are focused on high precision particle detectors. He is also involved in the construction of detectors for beam trajectory definition for hadron therapy facilities.

Sébastien Jiguet works at Gersteltec Sàrl, Switzerland, where he manages the R\&D department. He holds a MEng in multimaterials \& interfaces and a MSc in composite materials \& biomaterials from the University of Lyon, France, and completed his doctoral studies in Ès Sciences Techniques from the Ecole Poytechnique Fédérale de Lausanne (EPFL), Lausanne, Switzerland.

Giovanna Lehmann Miotto obtained the diploma in physics at the University of Milano, Italy, in 1996, and the PhD at the University of Bern, Switzerland, in 2000. She is involved in the field of high energy particle physics with a main focus in dataacquisition and trigger systems and is a staff at the European centre for particle physics (CERN), Geneva, Switzerland.

Wainer Vandelli is an experimental particle physicist and a staff member of the European centre for particle physics (CERN), Geneva, Switzerland. Since 2002 he is part of the ATLAS collaboration, where he contributes to the development and operation of the data-acquisition system. Previously, with the University of Pavia, Italy and INFN-Pavia, Italy he participated to the construction and validation of ATLAS muon detectors and to studies of the SUSY discovery potential at the LHC. In 2005 he spent 6 months in LBNL, Berkeley as a visiting researcher, working on a LHC luminosity monitor prototype. Presently he is also part of a dual-readout calorimetry R\&D collaboration (DREAM). In 2007, he obtained a PhD in Particle Physics from the University of Pavia, Italy.

Noelia Vico Triviño received her BSc in Physics and MSc in electronic engineering from the University of Granada, Spain, in 2007 and 2009, respectively. She carried out her MSc project in the Laboratoire de Microsystèmes (LMIS4) at the Ecole Polytechnique Fédérale de Lausanne (EPFL), Switzerland in 2009. She currently pursues her PhD in photonics at the Laboratory of Advanced Semiconductors for Photonics and Electronics (LASPE) at EPFL. Her research is focused on quantum dots and nanowires grown by molecular beam epitaxy (MBE).

Philippe Renaud was born in 1958. He received the diploma in physics from the University of Neuchatel, Neuchatel, Switzerland, in 1983, and the PhD degree from the University of Lausanne, Lausanne, Switzerland, in 1988. He was involved in the theoretical and experimental study of magnetoelastic effects. During 1988-1989, he was a postdoctoral researcher at the University of California, Berkeley. From 1990 to 1991, he was a postdoctoral researcher at the IBM Zurich Research Laboratory. In 1992, he joined the Sensors and Actuators Group, Swiss Center for Electronics and Microtechnology (CSEM), Neuchatel, Switzerland. In 1993, he became an assistant professor at the Swiss Federal Institute of Technology Lausanne (EPFL), Lausanne, where he was appointed a full professor in 1997 and is the director of the Center of MicroNanoTechnology (CMI), one of the largest state-of-the-art academic clean room facilities in the world, with processing equipment for training and scientific purposes in microelectronic and microfabrication processes. In the summer of 1996, he was a visiting professor at Tohoku University, Japan. His current research interests include microsystem design, microfabrication technologies for microelectromechanical system (MEMS) applications, microfluidics, and bio-MEMS applications. Prof. Renaud is the Chairman of the International Nanotech-Conference on Micro and Nanotechnologies for the Biosciences held annually in Montreux, Switzerland. 